\title{
Understanding Rigidity in Colloidal Gels
}

\author{
Correlated rigidity percolation and colloidal gels
}

Authors: Shang Zhang, Leyou Zhang, Mehdi Bouzid, D. Zeb Rocklin, Emanuela Del

Gado, and Xiaoming Mao

Phys. Rev. Lett. 123, 33 (2019)

\section{Recommended with a Commentary by Bulbul Chakraborty, Martin Fisher School of Physics, Brandeis University, Waltham, MA 02454}

Glasses and gels are the two paradigmatic examples of dynamically arrested, disordered solids. In contrast to glasses, gels in attractive colloids can form at relatively low densities $[1,2]$. Quoting from [1]: "Gels are amorphous materials composed of a liquid phase and an interconnected network like solid phase, which can be formed at very low solid volume fractions. They are widespread, both in nature and in industry - from the pharmaceutical industry (agar gel) to the construction sector (cement hydrate or aluminosilicate gels in cement) - or in everyday life: the bread gets its rubbery texture from the formation of an elastic network of gluten protein, and the formation of protein networks is vital for the successful production of cheese. All soft matter, in fact, from proteins to colloids and polymers easily self-assemble into such weakly elastic solids. They generally present a heterogeneous structure which is the result of the coupling between the kinetics of aggregation, phase separation or demixing, and arrest of the microscopic dynamics".

Unlike crystalline solids, where elasticity emerges as a consequence of broken symmetry, the origin of rigidity in amorphous solids is far from clear. Rigidity of gels, which occur at low densities is even harder to understand than the rigidity of glasses, which occur at high densities. Low-density gels are characterized by sparse networks of colloidal particles, which are sustained through attractive interactions. This geometric percolation is certainly necessary for gelation but it cannot be a sufficient condition for the emergence of mechanical rigidity. For covalently bonded networks, such as polymer gels, the idea that not all parts of a connected network are necessarily rigid is well established [3]. In contrast, discussion of colloidal gels have largely focussed on geometric percolation, with the assumption that all persistent clusters are rigid. The relation between mechanical rigidity and the connectivity of the gel network has received less attention.

The distinctive feature of a solid is its resistance to shear. The challenge faced in defining and computing the shear modulus of an amorphous solid was laid out succinctly in a seminal paper on rubber elasticity by Deam and Edwards [4]. They presented an elegant statistical mechanics approach that relied on the concept of conserved topologies to compute differences in free energies between sheared and unsheared states. An alternative to this approach, that 
has proven to be extremely useful in analyzing mechanical rigidity of disordered networks in dynamically arrested states is that of rigidity percolation [5]. As in any percolation problem, the objective is to identify connected pathways. In rigidity percolation, the paths are defined by sites that are mutually rigid, where rigid sites are identified through counting degrees of freedom and constraints. Unlike connectivity, rigidity is inherently a nonlocal property and thus whether a region of a network is floppy or rigid generally depends upon structural details that are far away. The onset of rigidity of a network is identified with the emergence of a system-spanning cluster of mutually rigid sites.

Since mechanical rigidity requires the formation of a mechanically stable spanning cluster that can transmit stresses, it is expected to and usually occurs at densities higher than those associated with geometric percolation. How does one reconcile this aspect of rigidity percolation with the appearance of mechanically rigid structures in suspensions of colloidal particles at volume fractions of just a few percent? This is the puzzle that is addressed in the paper by Zhang et al. The resolution of this puzzle is elegant and appealing. The central thesis of the paper is that the dynamical arrest responsible for gelation is a process of self-organization. The arrested structures are special in that they accommodate the local and global constraints of mechanical equilibrium: there are significant structural correlations, induced for example by attractive interactions, that need to be incorporated into any rigidity percolation analysis. In resolving the puzzle, this paper also highlights the need to link connectivity percolation, normally associated with gelation, with rigidity percolation and not view them as independent processes.

The authors support their hypothesis of mechanical rigidity in gels arising from correlated rigidity percolation by analyzing a lattice model, where structural correlations are built in through site occupation probabilities that depend on the surroundings [6, 7], and a microscopic model of an attractive gel where structural correlations appear as a consequence of the attraction. The idea that correlations can lead to special structures that occupy very little space yet are mechanically rigid is intuitive. The authors illustrate this through their example of a Warren truss, which is a special one-dimensional structure embedded in two dimensions. What the analysis in the paper demonstrates is that such structures can arise naturally in attractive colloids, and in the lattice model, as correlations are introduced. Increasing correlations either through decreasing temperature (increasing attraction strength), or explicitly in the lattice model leads to lowering of the volume fraction at which rigidity percolates. The phase diagrams presented in the paper convincingly demonstrate that the sparse networks that emerge during the process of dynamical arrest in attractive colloids are particularly well-equipped to transmit mechanical stresses. The fact that these structures are special is highlighted through the comparison to the lattice model.

By extending the classical rigidity percolation framework to include structural correlations, the authors have provided a new perspective on colloidal gelation. They have provided tools that can be used to further explore the interplay between local rigidity in clusters and dynamic slowing down in the self-organization process that leads to mechanical rigidity of dynamically arrested states. These tools should also be helpful in aiding the design of novel ultralight materials that are mechanically rigid, and carry stress. In addition, they introduce the idea of correlated percolation, which was known in the context of connectivity percolation $[6,7]$, to rigidity percolation. 


\section{References}

[1] M. Bouzid and E. Del Gado, in Mechanics of Soft Gels, Handbook of Materials Modeling: Applications: Current and Emerging Materials, edited by W.Andreoni and S. Yip (Springer, Cham, 2019).

[2] E. Del Gado, D. Fiocco, G. Foffi, S. Manley, V. Trappe, and A. Zaccone, in Fluids, Colloids and Soft Materials: An Introduction to Soft Matter Physics (John Wiley \& Sons, Hoboken, 2016), p. 279

[3] P. G. deGennes, Scaling Concepts in Polymer Physics (Cornell University Press, Ithaca, N.Y., 1979)

[4] R. T. Deam and S. F. Edwards, Proc Phil Trans 280, 317-353, 1976

[5] D. J. Jacobs and M. F. Thorpe, Phys. Rev. Lett. 75, 4051 (1995)

[6] A. Coniglio, J. Phys. A: Math. Phys. 12, 545 (1979)

[7] A. Coniglio, H. E. Stanley, and W. Klein, Phys. Rev. Lett. 42, 518 (1979) 